\title{
Is there ageism in prostate cancer detection?
}

\author{
Neil Fleshner, MD, MPH, FRCSC
}

See related article on page 205

\section{$\mathrm{T}$}

here has been continuing controversy regarding the role of prostate-specific antigen (PSA) testing in the diagnosis of prostate cancer, particularly in the screening scenario. Recent data from the New England Journal of Medicine suggest that mortality related to prostate cancer can be reduced at 8.8 years after diagnosis by about $20 \%$ among a cohort of men between 55 and 74 years of age. ' What are we to do with the men 75 and older?

As pointed out by Mistry and colleagues, ${ }^{2}$ life expectancy among men continues to increase. Furthermore, men are more physically and sexually active as they age, compared with 20 and 30 years ago. The morbidity and mortality of cardiovascular diseases are also declining. How are we to incorporate these changing realities into the detection of prostate cancer among elderly men?

Mistry and colleagues reviewed the results of 1446 needle biopsies among men aged 75 and older. Not surprisingly, $52 \%$ of the biopsies were positive for prostate cancer and $78 \%$ of those would be defined as clinically significant by current methodology. The authors conclude that there may be a benefit in aggressively pursuing a prostate cancer diagnosis among men older than 75.

There are many limitations to the authors' study that must be clarified. First, there is no information, as acknowledged by the authors, about the indication for biopsy among these men older than 75 years. It is very possible that it was not PSA but significant findings on digital rectal examination that drove these biopsies. Clearly, as men age their risk of high-grade disease increases and, indeed, my research group has shown this in some of our previous analyses. ${ }^{3}$ Furthermore, we know that men with significant prostate nodules are at a higher risk for clinically significant disease. ${ }^{4}$ In this sense, the clinical findings in this article are not particularly novel.

Second, another significant limitation continues to be our definition of "significant prostate cancer." The original definition of significant prostate cancer was derived from radical cystoprostatectomy specimens and the features of prostate cancers therein. A rank analysis was then done taking the worse percentile of patients and using these as features to predict death from prostate cancer. There are obvious flaws in this methodology that we have been stuck with for many years.

From a patient's point of view, however, clinically significant prostate cancer represents prostate cancer that if untreated would cause undue morbidity and/or mortality for the patient. Since the prostate cannot be removed, examined and replaced into the body, it remains extremely difficult to define what a clinically significant cancer is. Nevertheless, the vast majority of nonmetastatic prostate cancer does not impact on longevity within a 0-7 year window. In addition, the vast majority of these cases would unlikely affect mortality for 12-20 years.

Given these truisms and some of the methodological limitations of the article by Mistry and colleagues, I do not believe that current practice should change. Should we continue to order biopsies for men older than 75 years? Of course the answer is "Yes." If a man has an abnormal finding on digital rectal examination or if his PSA level is significantly high, then a prostate biopsy is warranted and, indeed, treatment for that prostate cancer may be warranted. Keep in mind, however, that randomized data suggest no benefit to radical prostatectomy in terms of survival for men older than 65 years. ${ }^{5}$ These data of course may not apply to men with more advanced disease, and modalities such as radiotherapy and hormonal therapy remain viable options.

Should we provide annual PSA testing for men older than 75 ? In my opinion, the answer is "No." The prevalence of prostate cancer that is indolent, or even what we would consider on histology clinically "significant," and would have no impact on the patient, is far too high to warrant this. Furthermore, the burden of overtreatment, particularly in the forms of radical therapy or hormonal therapy, have a particular toll on elderly patients. In my view, the risk-benefit analysis simply does not justify this.

Does this mean that ageism exists in the detection of prostate cancer among elderly men? No. It is simply a product of the fact that as men get older, the number of years they have to live is on average less than it is for younger men. This is coupled with the increase in the morbidity of treatment. I am a strong proponent of PSA screening and testing, 
but I believe we would do greater harm than benefit if we were to employ this among men older than 75 years.

Head, Division of Urology, Princess Margaret Hospital, University Health Network, Toronto, Ont. Competing interests: None declared.

\section{References}

1. Schröder FH, Hugosson J, Roobol MJ, et al.; ERSPC Investigators. Screening and prostate-cancer mortality in a randomized European study. N Engl J Med 2009;360:1320-8.
2. Mistry S, Mayer W, Khavari R, et al. Who's too old to screen? Prostate cancer in elderly men. Can Urol Assoc J 2009:3:205-10.

3. Kulkarni GS, Al-Azab R, Lockwood G, et al. Evidence for a biopsy derived grade artiffact among larger prostate glands. J Urol 2006; 175:505-9.

4. Toi A, Neill MG, Lockwood GA, et al. The continuing importance of transrectal ultrasound identification of prostatic lesions. J Urol 2007;177:516-20.

5. Bill-Axelson A, Holmberg L, Ruutu M, et al.; Scandinavian Prostate Cancer Group Study No. 4. Radical prostatectomy versus watchful waiting in early prostate cancer. N Engl J Med 2005;352:1977-84.

Correspondence: Dr. Neil Fleshner, 610 University Ave., Suite 3-130, Toronto ON M5G 2M9; neil.fleshner@uhn.on.ca

\section{Alberta Health
Services \\ Urologist}

The Alberta Health Services - Calgary, Department of Surgery invites applications for a Urologist to work in the Rockyview Hospital, one of three adult acute care centres in Calgary, Alberta. The candidate's clinical expertise should enrich the Division of Urology.

The successful candidate will become a member of the Division of Urology and provide urological care in the Calgary area. Duties will include patient care to the community of Calgary and surrounding areas. Teaching at the undergraduate and post graduate level will be required. Committee duties will be expected as the need arises. Successful applicants will take call according to the site and regional policies.

Qualifications include an MD, a Fellowship of the Royal College of Physicians and Surgeons of Canada in Urology with additional experience in Oncology and Laparoscopic Surgery and eligibility for licensure in the province of Alberta. In accordance with Canadian immigration requirements, priority will be given to Canadian citizens and permanent residents of Canada. Alberta Health Services is committed to employment equity.

The successful individual will be expected to have a clinical appointment with the University of Calgary, Faculty of Medicine, Department of Surgery and to participate in medical student and resident teaching.

Please submit curriculum vitae and the names and addresses of three referees by June 30, 2009 to:

$$
\text { Dr. John Dushinski }
$$

Regional Clinical Division Chief

Division of Urology, Department of Surgery \#210, 1011 Glenmore Trail SW

$$
\text { Calgary, AB T2V 4R6 }
$$

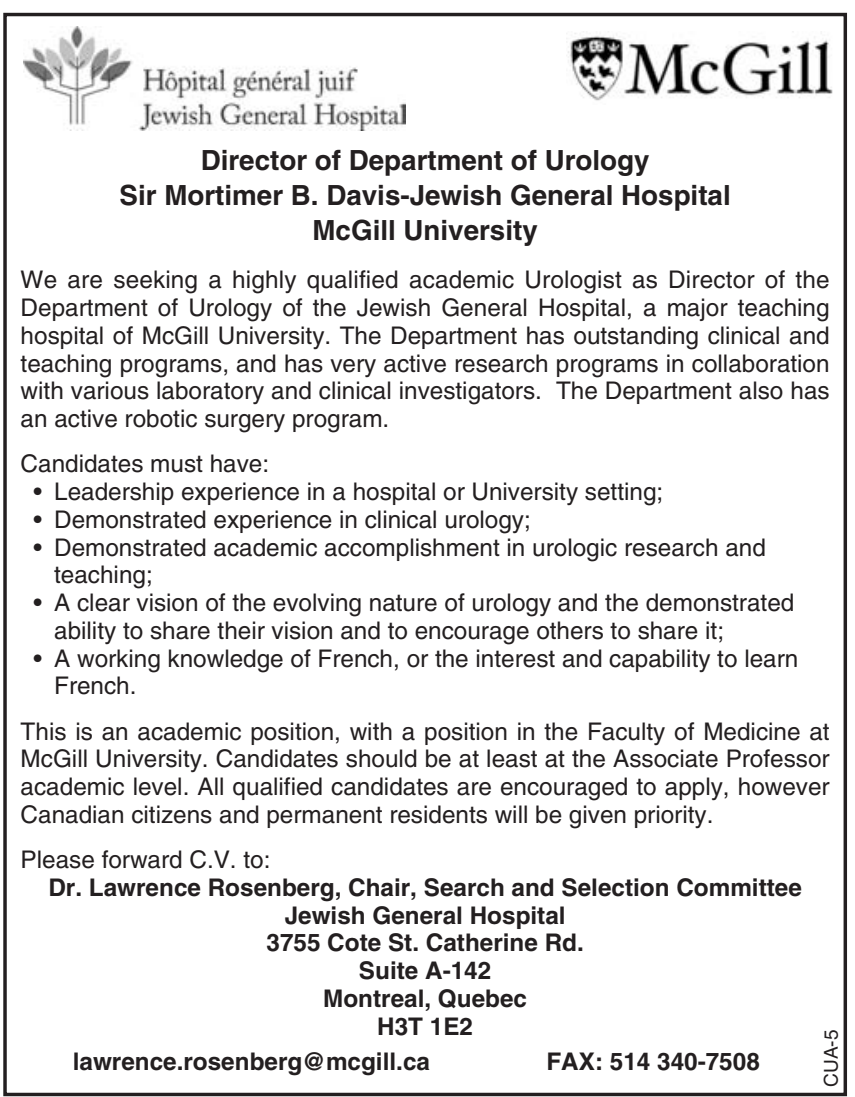

